\title{
(Inter)subjectivisation et chaines sémantiques dans les adverbes français en -ment : analyse du passage de l'adverbe intégré à la proposition au marqueur discursive- CORRIGENDUM
}

\section{Emma Álvarez-Prendes}

https://doi.org/10.1017/cnj.2021.23. Published by Cambridge University Press, 3 August 2021

In the following passage of the article by Álvarez-Prendes (2021), the year was omitted from a citation of an article by Heine et al. (1991). The citation should appear as follows:

Il ne reste à présent qu'à examiner les mécanismes linguistiques grâce auxquels ces processus ont été accomplis. L'analyse diachronique de nos huit opérateurs a révélé des parcours sémantiques relativement stables, ainsi que dégagé un certain nombre de chaines sémantiques (Heine et al. 1991, Heine et Kuteva 2002).

This correction has been made.

\section{RÉFÉRENCES}

Álvarex-Prendes, Emma. Published online 2021. (Inter)subjectivisation et chaines sémantiques dans les adverbes français en -ment : analyse du passage de l'adverbe intégré à la proposition au marqueur discursive. Canadian Journal of Linguistics/Revue canadienne de linguistique. https://doi.org/10.1017/cnj.2021.23.

Heine, Bernd, Ulrike Claudi et Friederike Hünnemeyer. 1991. Grammaticalization. A conceptual framework. Chicago : The University of Chicago Press.

Heine, Bernd et Tania Kuteva. 2002. World lexicon of grammaticalization. Cambridge: Cambridge University Press. 